\title{
Minimal polynomials of some beta-numbers and Chebyshev polynomials
}

\author{
by \\ DoYong KWON (Seoul)
}

1. Introduction. For $\beta>1$, the $\beta$-transformation $T_{\beta}: x \mapsto \beta x(\bmod 1)$ is multiplication by $\beta$ modulo 1 . Then the $\beta$-expansion $d_{\beta}(x)=\left(x_{i}\right)_{i \geq 1}$ of $x \in[0,1]$ is defined by $x_{i}=\left\lfloor\beta T_{\beta}^{i-1}(x)\right\rfloor$. While Rényi [15] introduced the $\beta$-transformation and proved that it is ergodic, Parry [13] found its invariant measure and characterized possible sequences that can be a $\beta$-expansion. It was shown that the $\beta$-expansion of 1 bounds, in a sense, the $\beta$-expansion of any other $x \in[0,1)$ in terms of lexicographic order. To be more precise, let us fix $\beta>1$ and assume that $s=x_{1} x_{2} \cdots$ is an infinite word over the alphabet $\{0,1, \ldots,\lfloor\beta\rfloor\}$. In addition, put

$$
d_{\beta}^{*}(1):=\lim _{\varepsilon \backslash 0} d_{\beta}(1-\varepsilon)
$$

we have $d_{\beta}^{*}(1)=\left(e_{1} \cdots e_{m-1}\left(e_{m}-1\right)\right)^{\omega}$ if $d_{\beta}(1)=e_{1} \cdots e_{m} 0^{\omega}$ and $d_{\beta}^{*}(1)=$ $d_{\beta}(1)$ otherwise. Here $a^{\omega}:=a a \cdots$. Then Parry showed that $s=d_{\beta}(x)$ for some $x \in[0,1)$ if and only if $\sigma^{n}(s)<d_{\beta}^{*}(1)$ for all $n \geq 0$, where $\sigma$ is the shift.

If $d_{\beta}(1)$ is eventually periodic, say $d_{\beta}(1)=e_{1} \cdots e_{n}\left(e_{n+1} \cdots e_{n+p}\right)^{\omega}$, then we call $\beta$ a beta-number. In this case, $\beta$ is the dominant root of

$$
\left(x^{n+p}-\sum_{i=1}^{n+p} e_{i} x^{n+p-i}\right)-\left(x^{n}-\sum_{i=1}^{n} e_{i} x^{n-i}\right)=0 .
$$

In particular, if $d_{\beta}(1)$ is finite, i.e., $d_{\beta}(1)=e_{1} \cdots e_{n} 0^{\omega}$, then we call $\beta$ a simple beta-number and $\beta$ is the dominant root of

$$
x^{n}-\sum_{i=1}^{n} e_{i} x^{n-i}=0 .
$$

2000 Mathematics Subject Classification: Primary 11R06, 11R09; Secondary 37B10, $68 \mathrm{R} 15$

Key words and phrases: beta-numbers, Chebyshev polynomials, Pisot numbers, Mahler measure. 
These two polynomials above are called the beta-polynomial of $\beta$, or simply the $\beta$-polynomial if $\beta$ is clear from the context. The term characteristic polynomial is also used in the literature.

We focus on a special class of $\beta$-expansions. We study the real $\beta>1$ for which $d_{\beta}^{*}(1)$ encodes a rational rotation on $\mathbb{R} / \mathbb{Z}$. In this case, $\beta$ is an algebraic integer. For the case of irrational rotations, see $[4,6]$. An arithmetic study was also pursued there and the current work is in fact motivated by those papers.

Let $\alpha>0$ and $\varrho \in[0,1]$. We consider the following two infinite sequences called lower and upper mechanical words with slope $\alpha$ and intercept $\varrho$ : for $n \geq 0$,

$$
\begin{aligned}
& s_{\alpha, \varrho}(n)=\lfloor\alpha(n+1)+\varrho\rfloor-\lfloor\alpha n+\varrho\rfloor, \\
& s_{\alpha, \varrho}^{\prime}(n)=\lceil\alpha(n+1)+\varrho\rceil-\lceil\alpha n+\varrho\rceil .
\end{aligned}
$$

Note that these are infinite words over the alphabet $A=\{\lceil\alpha\rceil-1,\lceil\alpha\rceil\}$. For an irrational $\alpha$, the words are aperiodic and called Sturmian words. On the other hand, a rational $\alpha$ produces purely periodic words, whose shortest period words are called Christoffel words. Our main concern is the rational case. We now describe it in more detail.

Let $\alpha=p / q>0$ with $\operatorname{gcd}(p, q)=1$ and $b=\lceil\alpha\rceil$. Then $A=\{b-1, b\}$ and the lengths of the Christoffel words, say $t_{p, q}, t_{p, q}^{\prime}$, are $q$. So $s_{\alpha, 0}=t_{p, q}^{\omega}$ and $s_{\alpha, 0}^{\prime}=\left(t_{p, q}^{\prime}\right)^{\omega}$. We also have

$$
t_{p, q}=(b-1) z_{p, q} b, \quad t_{p, q}^{\prime}=b z_{p, q}(b-1),
$$

for some word $z_{p, q}$, called a central word. We see that $z_{p, q}$ is a palindrome, i.e., $z_{p, q}$ is equal to its reversal (see [12]). The motivation of this paper comes from the next proposition. For any word $w=a_{0} a_{1} \cdots a_{n-1}$ with $a_{i} \in \mathbb{Z}$, we denote by $\vec{w}$ the vector $\left(a_{0}, \ldots, a_{n-1}\right) \in \mathbb{Z}^{n}$.

Proposition $1.1([4,6])$. For $\alpha>0$, there exists a unique $\beta>1$ for which $d_{\beta}^{*}(1)=s_{\alpha, 0}^{\prime}$. Define $\Delta:(0, \infty) \rightarrow(1, \infty)$ by $\Delta(\alpha)=\beta$. Then

(a) At an irrational $\alpha>0, \Delta$ is continuous, and $\Delta(\alpha)$ is a transcendental number.

(b) $\Delta$ is left-continuous but not right-continuous at every rational.

(c) If $\alpha=p / q$ with $\operatorname{gcd}(p, q)=1$ and $b=\lceil\alpha\rceil$, then the $\Delta(\alpha)$-polynomial is

$$
x^{q}-\overrightarrow{b z_{p, q} b} \cdot\left(x^{q-1}, x^{q-2}, \ldots, 1\right) .
$$

(d) For the same rational $\alpha$ as above, let $\Delta(\alpha+):=\lim _{x \rightarrow \alpha+} \Delta(x)$. Then the $\Delta(\alpha+)$-polynomial is

$$
x^{q+1}-\overrightarrow{b z_{p, q} b} \cdot\left(x^{q}, x^{q-1}, \ldots, x\right)-x+1 .
$$


For a rational $\alpha>0, \Delta(\alpha)$ is called a lower self-Christoffel number, and the right limit $\Delta(\alpha+)$ an upper self-Christoffel number.

We are now in a position to state our main theorem.

Theorem. Let $p_{0} / q_{0}$ be a fixed rational with $0<p_{0} \leq q_{0}$ and $\operatorname{gcd}\left(p_{0}, q_{0}\right)$ $=1$. For a positive integer $b$, suppose $p / q=b-1+p_{0} / q_{0}$. Then there exists an effectively computable $B$ such that for all $b \geq B$, we have:

(a) $x^{q}-\overrightarrow{b z_{p, q} b} \cdot\left(x^{q-1}, x^{q-2}, \ldots, 1\right)$ is irreducible over $\mathbb{Q}$,

(b) $x^{q+1}-\overrightarrow{b z_{p, q} b} \cdot\left(x^{q}, x^{q-1}, \ldots, x\right)-x+1$ is irreducible over $\mathbb{Q}$.

Thus the polynomials mentioned are eventually the minimal polynomials of $\Delta(p / q)$ and $\Delta(p / q+)$. The above theorem was in fact proved in [7] but we did not know from which value $b$ on the polynomials were irreducible. Here we give another proof involving Chebyshev polynomials. The new proof enables us to effectively find $B$ for which $b \geq B$ implies that the corresponding polynomials are irreducible over $\mathbb{Q}$. The effective procedure for finding $B$ is given in Section 4. These quantitative results have some connections with other fields of number theory, which will be discussed in Section 4.

2. Preliminaries. In this section we briefly review some concepts and known results to be used in the main proof. First we recall some definitions in number theory.

Among algebraic integers, a Pisot number is $\alpha>1$ all of whose conjugates lie inside the unit circle. Suppose $g(x)=a_{n} x^{n}+\cdots+a_{1} x+a_{0}=$ $a_{n} \prod_{i=1}^{n}\left(x-\alpha_{i}\right) \in \mathbb{Z}[x]$ with $a_{n} \neq 0$. Then the Mahler measure of $g$ is the positive number defined by

$$
M(g)=\left|a_{n}\right| \prod_{i=1}^{n} \max \left\{1,\left|\alpha_{i}\right|\right\} .
$$

In particular, cyclotomic polynomials have Mahler measure 1. The most famous problem on Mahler measures, posed by Lehmer [10], is whether or not 1 is an accumulation point of the set $\{M(g) \mid g \in \mathbb{Z}[x]\}$. Though it is still open, an answer can be given for a special class of polynomials. Let $f \in \mathbb{R}[x]$ and $\operatorname{deg}(f)=n$. If $f$ satisfies $f(x)=x^{n} f\left(x^{-1}\right)$ then we say that $f$ is reciprocal. Now the Mahler measures of nonreciprocal polynomials cannot be less than the smallest Pisot number:

Theorem 2.1 ([17]). Let $p(x) \in \mathbb{Z}[x]$ and let $\theta_{0}=1.32472 \ldots$ be the smallest Pisot number, i.e., the real zero of $x^{3}-x-1$. If $M(p)<\theta_{0}$, then $p(x)$ is a reciprocal polynomial.

Let $T_{n}$ and $U_{n}$ be the $n$th Chebyshev polynomials of the first and the second kind respectively. Among many equivalent definitions we adopt the 
following simple equations, which are suitable for our proof:

$$
T_{n}(\cos \theta):=\cos n \theta, \quad U_{n}(\cos \theta):=\frac{\sin (n+1) \theta}{\sin \theta} .
$$

For instance, $T_{2}(x)=2 x^{2}-1$ and $U_{3}(x)=8 x^{3}-4 x$.

In the analysis of our polynomials, we shall encounter some reciprocal polynomials, and they will be converted into more convenient ones via a transformation introduced in [3].

Suppose that $p(z)=\sum_{i=0}^{2 n} a_{i} z^{i} \in \mathbb{R}[z]$ is a nonzero reciprocal polynomial with $a_{i}=a_{2 n-i}, i=0, \ldots, n$. If $a_{2 n}=a_{2 n-1}=\cdots=a_{n+k+1}=0$ but $a_{n+k} \neq 0$, then

$$
\begin{aligned}
p(z) & =\sum_{i=0}^{2 n} a_{i} z^{i}=z^{n}\left[a_{n+k}\left(z^{k}+\frac{1}{z^{k}}\right)+\cdots+a_{n+1}\left(z+\frac{1}{z}\right)+a_{n}\right] \\
& =a_{n+k} z^{n} \prod_{i=1}^{k}\left(z+\frac{1}{z}-\alpha_{i}\right)=a_{n+k} z^{n-k} \prod_{i=1}^{k}\left(z^{2}-\alpha_{i} z+1\right)
\end{aligned}
$$

for some $\alpha_{i} \in \mathbb{C}, i=1, \ldots, k$. Given a reciprocal polynomial $p$ as above, the Chebyshev transform $\mathcal{T}$ of $p$ is defined by

$$
\mathcal{T} p(x):=a_{n+k} \prod_{i=1}^{k}\left(x-\alpha_{i}\right) .
$$

TheOREM $2.2([3])$. The Chebyshev transform $\mathcal{T}$ is a linear isomorphism of the space of real reciprocal polynomials, $\left\{p(z)=\sum_{i=0}^{2 n} a_{i} z^{i} \in \mathbb{R}[z]: a_{i}=\right.$ $\left.a_{2 n-i}, i=0, \ldots, n\right\}$, into the space of real polynomials of degree at most $n$.

Note that

$$
\left|p\left(e^{i \theta}\right)\right|=|\mathcal{T} p(2 \cos \theta)| .
$$

Chebyshev polynomials now naturally appear via Chebyshev transforms.

Lemma 2.3 ([9]). If $p(z)=z^{2 n}+1$ and $q(z)=z^{2 n}+z^{2 n-2}+\cdots+z^{2}+1$, then $\mathcal{T} p(x)=2 T_{n}(x / 2)$ and $\mathcal{T} q(x)=U_{n}(x / 2)$.

The following geometric study on beta-polynomials will be useful below. The reader should note that all the bounds given in the proposition tend to 1 as $\beta$ increases.

\section{Proposition 2.4 ([7]).}

(a) If $\beta$ is an upper self-Christoffel number and $\gamma \neq \beta$ is a zero of the $\beta$-polynomial, then

$$
|\gamma| \leq \frac{\beta+\sqrt{\beta^{2}+4 \beta}}{2 \beta}
$$


(b) If $\beta$ is a lower self-Christoffel number and $\gamma \neq \beta$ is a zero of the $\beta$-polynomial, then

$$
\frac{2 \beta+1-\sqrt{8 \beta+1}}{2 \beta} \leq|\gamma| \leq \frac{2 \beta+1+\sqrt{8 \beta+1}}{2 \beta} .
$$

3. Proof. Throughout this section, the notations appearing in the Theorem will be used without explicit mention. First we consider lower selfChristoffel numbers.

Put $f(x)=x^{q}-\overrightarrow{b z_{p, q} b} \cdot\left(x^{q-1}, x^{q-2}, \ldots, 1\right)$ and suppose that $f(x)=$ $g(x) h(x)$ over $\mathbb{Q}$ and $g(\beta) \neq 0=h(\beta)$. Then Theorem 2.1 together with Proposition 2.4 implies that $g$ is eventually reciprocal as $b$ increases. If $\gamma$ is a zero of $g$, then so is $\gamma^{-1}$. Thus

$$
\begin{aligned}
\gamma^{q} & =\overrightarrow{b z_{p, q} b} \cdot\left(\gamma^{q-1}, \gamma^{q-2}, \ldots, 1\right)=\gamma^{q-1} \overrightarrow{b z_{p, q} b} \cdot\left(\gamma^{1-q}, \gamma^{2-q}, \ldots, 1\right) \\
& =\gamma^{q-1} \gamma^{-q}=\gamma^{-1},
\end{aligned}
$$

where we use the fact that $b z_{p, q} b$ is a palindrome. So $\gamma^{q+1}=1$.

Assume that $q=2 n+1$ and $\gamma_{j}=e^{i \theta_{j}}=e^{2 j \pi i /(q+1)}$ is a zero of $g$ for some $j=1, \ldots, q$. We then consider the polynomial

$$
\begin{aligned}
r(z) & :=z^{q}-f(z)=\overrightarrow{b z_{p, q} b} \cdot\left(z^{2 n}, z^{2 n-1}, \ldots, 1\right) \\
& =b\left(z^{2 n}+z^{2 n-1}+\cdots+1\right)-\sum_{k=1}^{n} a_{k} z^{k}\left(z^{2 n-2 k}+1\right), \quad a_{k} \in\{0,1 / 2,1\} .
\end{aligned}
$$

Together with the equation

$$
z^{2 n}+z^{2 n-1}+\cdots+1=\left(z^{2 n}+z^{2 n-2}+\cdots+z^{2}+1\right)+z\left(z^{2 n-2}+z^{2 n-4}+\cdots+z^{2}+1\right),
$$

Lemma 2.3 guarantees that the Chebyshev transform of $r(z)$ is

$$
\mathcal{T} r(x)=b\left(U_{n}\left(\frac{x}{2}\right)+U_{n-1}\left(\frac{x}{2}\right)\right)-\sum_{k=1}^{n} 2 a_{k} T_{n-k}\left(\frac{x}{2}\right) .
$$

To compute $\operatorname{Tr}\left(2 \cos \theta_{j}\right)$, we use the fact that $T_{n-k}\left(\cos \theta_{j}\right)=\cos (n-k) \theta_{j}$ and that

$$
\begin{aligned}
U_{n}\left(\cos \theta_{j}\right)+U_{n-1}\left(\cos \theta_{j}\right) & =\frac{\sin (n+1) \theta_{j}+\sin n \theta_{j}}{\sin \theta_{j}} \\
& =\frac{2 \sin \frac{(2 n+1) \theta_{j}}{2} \cos \frac{\theta_{j}}{2}}{\sin \theta_{j}}=\frac{\sin \frac{(2 n+1) \theta_{j}}{2}}{\sin \frac{\theta_{j}}{2}} .
\end{aligned}
$$

Thus one finds that

$$
1=\left|\mathcal{T} r\left(2 \cos \theta_{j}\right)\right| \geq b \frac{\left|\sin \frac{(2 n+1) \theta_{j}}{2}\right|}{\left|\sin \frac{\theta_{j}}{2}\right|}-\sum_{k=1}^{n} 2 a_{k} .
$$


Since $0<\theta_{j} / 2<\pi$ and $(2 n+1) \theta_{j} / 2=(2 n+1) j \pi /(2 n+2)$ cannot be an integer multiple of $\pi$ for any $j=1, \ldots, 2 n+1$, this leads to a contradiction as $b$ increases.

If $q=2 n$, then

$$
\begin{aligned}
r(z) & =z^{q}-f(z)=\overrightarrow{b z_{p, q} b} \cdot\left(z^{2 n-1}, z^{2 n-2}, \ldots, 1\right) \\
& =b\left(z^{2 n-1}+z^{2 n-2}+\cdots+1\right)-\sum_{k=1}^{n-1} a_{k} z^{k}\left(z^{2 n-2 k-1}+1\right), \quad a_{k} \in\{0,1\} .
\end{aligned}
$$

If $n=1$, then the sum is understood to be zero. Define a polynomial $r_{1}(z)$ by

$$
\begin{aligned}
r_{1}(z):=\frac{r(z)}{z+1}= & b\left(z^{2 n-2}+z^{2 n-4}+\cdots+z^{2}+1\right) \\
& -\sum_{k=1}^{n-1} a_{k} z^{k}\left(z^{2 n-2 k-2}-z^{2 n-2 k-3}+\cdots-z+1\right) .
\end{aligned}
$$

Its Chebyshev transform is

$$
\mathcal{T} r_{1}(x)=b U_{n-1}\left(\frac{x}{2}\right)-\sum_{k=1}^{n-1} a_{k}\left(U_{n-k-1}\left(\frac{x}{2}\right)-U_{n-k-2}\left(\frac{x}{2}\right)\right) .
$$

If $\gamma_{j}=e^{i \theta_{j}}=e^{2 j \pi i /(q+1)}$ is a zero of $g$ for some $j=1, \ldots, q$, then

$$
\begin{array}{r}
U_{n-k-1}\left(\cos \theta_{j}\right)-U_{n-k-2}\left(\cos \theta_{j}\right)=\frac{\sin (n-k) \theta_{j}-\sin (n-k-1) \theta_{j}}{\sin \theta_{j}} \\
=\frac{2 \cos \frac{(2 n-2 k-1) \theta_{j}}{2} \sin \frac{\theta_{j}}{2}}{\sin \theta_{j}}=\frac{\cos \frac{(2 n-2 k-1) \theta_{j}}{2}}{\cos \frac{\theta_{j}}{2}} .
\end{array}
$$

Since $\gamma_{j} \neq-1$, there is a constant $M$ so that

$$
M \geq\left|\mathcal{T} r_{1}\left(2 \cos \theta_{j}\right)\right| \geq b \frac{\left|\sin n \theta_{j}\right|}{\left|\sin \theta_{j}\right|}-\sum_{k=1}^{n} \frac{a_{k}}{\left|\cos \frac{\theta_{j}}{2}\right|} .
$$

For any $j=1, \ldots, 2 n$, the number $n \theta_{j}=2 n j \pi /(2 n+1)$ is never an integer multiple of $\pi$ and the number $\theta_{j} / 2=j \pi /(2 n+1)$ cannot be $\pi / 2$. Now we get a contradiction for every sufficiently large $b$. This proves part (a) of the Theorem.

The crucial ingredient in the previous proof is to split a given betapolynomial into a reciprocal polynomial and a monomial. This technique also works for upper self-Christoffel numbers.

Put $f(x)=x^{q+1}-\overrightarrow{b z_{p, q} b} \cdot\left(x^{q}, x^{q-1}, \ldots, x\right)-x+1$ and suppose that $f(x)=g(x) h(x)$ over $\mathbb{Q}$ and $g(\beta) \neq 0=h(\beta)$. Then the same reasoning as before shows that $g$ is eventually reciprocal as $b$ increases. If $\gamma$ is a zero of $g$, 
then so is $\gamma^{-1}$. Hence

$$
\begin{aligned}
\gamma & =\gamma^{q+1}-\overrightarrow{b z_{p, q} b} \cdot\left(\gamma^{q}, \gamma^{q-1}, \ldots, \gamma\right)+1 \\
& =\gamma^{q+1}\left[\gamma^{-q-1}-\overrightarrow{b z_{p, q} b} \cdot\left(\gamma^{-q}, \gamma^{-q+1}, \ldots, \gamma^{-1}\right)+1\right]=\gamma^{q+1} \gamma^{-1}=\gamma^{q}
\end{aligned}
$$

which gives $\gamma^{q-1}=1$.

First, we suppose that $q=2 n-1$ and $\gamma_{j}=e^{i \theta_{j}}=e^{2 j \pi i /(q-1)}$ is a zero of $g$ for some $j=1, \ldots, q-2$. We then put

$$
\begin{aligned}
r(z):= & f(z)+z=z^{2 n}-\overrightarrow{b z_{p, q} b} \cdot\left(z^{2 n-1}, z^{2 n-2}, \ldots, z\right)+1 \\
= & z^{2 n}+z^{2 n-1}+\cdots+1-(b+1) z\left(z^{2 n-2}+\cdots+1\right) \\
& +\sum_{k=1}^{n} a_{k} z^{k}\left(z^{2 n-2 k}+1\right), \quad a_{k} \in\{0,1 / 2,1\} .
\end{aligned}
$$

Similar arguments to those above yield the Chebyshev transform

$$
\begin{aligned}
\mathcal{T} r(x)= & U_{n}\left(\frac{x}{2}\right)+U_{n-1}\left(\frac{x}{2}\right)-(b+1)\left(U_{n-1}\left(\frac{x}{2}\right)+U_{n-2}\left(\frac{x}{2}\right)\right) \\
& +\sum_{k=1}^{n} 2 a_{k} T_{n-k}\left(\frac{x}{2}\right) .
\end{aligned}
$$

Using $T_{n-k}\left(\cos \theta_{j}\right)=\cos (n-k) \theta_{j}$ and (1) one thus finds that

$$
1=\left|\mathcal{T} r\left(2 \cos \theta_{j}\right)\right| \geq(b+1) \frac{\left|\sin \frac{(2 n-1) \theta_{j}}{2}\right|}{\left|\sin \frac{\theta_{j}}{2}\right|}-\frac{\left|\sin \frac{(2 n+1) \theta_{j}}{2}\right|}{\left|\sin \frac{\theta_{j}}{2}\right|}-\sum_{k=1}^{n} 2 a_{k} .
$$

Since $0<\theta_{j} / 2<\pi$ and $(2 n-1) \theta_{j} / 2=(2 n-1) j \pi /(2 n-2)$ cannot be an integer multiple of $\pi$ for any $j=1, \ldots, 2 n-3$, we eventually get a contradiction as $b$ increases.

If $q=2 n$, then

$$
\begin{aligned}
r(z)= & f(z)+z=z^{2 n+1}-\overrightarrow{b z_{p, q} b} \cdot\left(z^{2 n}, z^{2 n-1}, \ldots, z\right)+1 \\
= & z^{2 n+1}+z^{2 n}+\cdots+1-(b+1) z\left(z^{2 n-1}+\cdots+1\right) \\
& +\sum_{k=1}^{n} a_{k} z^{k}\left(z^{2 n+1-2 k}+1\right), \quad a_{k} \in\{0,1\} .
\end{aligned}
$$

This can be rewritten in the form

$$
\begin{aligned}
r_{1}(z):= & \frac{r(z)}{z+1} \\
= & z^{2 n}+z^{2 n-2}+\cdots+z^{2}+1-(b+1) z\left(z^{2 n-2}+z^{2 n-4}+\cdots+z^{2}+1\right) \\
& +\sum_{k=1}^{n} a_{k} z^{k}\left(z^{2 n-2 k}-z^{2 n-2 k-1}+\cdots-z+1\right) .
\end{aligned}
$$


Hence we find

$\mathcal{T} r_{1}(x)=U_{n}\left(\frac{x}{2}\right)-(b+1) U_{n-1}\left(\frac{x}{2}\right)+\sum_{k=1}^{n} a_{k}\left(U_{n-k}\left(\frac{x}{2}\right)-U_{n-k-1}\left(\frac{x}{2}\right)\right)$. If $\gamma_{j}=e^{i \theta_{j}}=e^{2 j \pi i /(q-1)}$ is a zero of $g$ for some $j=1, \ldots, q-2$, then one can verify as in (2) that

$$
U_{n-k}\left(\cos \theta_{j}\right)-U_{n-k-1}\left(\cos \theta_{j}\right)=\frac{\cos \frac{(2 n-2 k+1) \theta_{j}}{2}}{\cos \frac{\theta_{j}}{2}} .
$$

Since $\gamma_{j} \neq-1$, there is a constant $M$ so that

$$
M \geq\left|\mathcal{T} r_{1}\left(2 \cos \theta_{j}\right)\right| \geq(b+1) \frac{\left|\sin n \theta_{j}\right|}{\left|\sin \theta_{j}\right|}-\frac{\left|\sin (n+1) \theta_{j}\right|}{\left|\sin \theta_{j}\right|}-\sum_{k=1}^{n} \frac{a_{k}}{\left|\cos \frac{\theta_{j}}{2}\right|} .
$$

For any $j=1, \ldots, 2 n-2$, the number $n \theta_{j}=2 n j \pi /(2 n-1)$ is never an integer multiple of $\pi$ and the number $\theta_{j} / 2=j \pi /(2 n-1)$ cannot be $\pi / 2$. Now we get a contradiction for every sufficiently large $b$.

4. Discussion and further studies. Suppose that an integer $b \geq 1$ is divisible by a prime $p$ but not by $p^{2}$. Then the Eisenstein criterion shows that the beta-polynomial of $\Delta((b q-1) / q)$ is irreducible for any $q \geq 1$. Now we introduce another interesting connection between prime numbers and irreducibility for self-Christoffel numbers. This is related to Mersenne primes, i.e., primes of the form $2^{n}-1$. A famous open question in number theory is:

Are there infinitely many Mersenne primes?

We need a classical irreducibility criterion for polynomials in $\mathbb{Z}[x]$, which appears for example in [14].

TheOREM 4.1. Let $\beta_{1}, \ldots, \beta_{n}$ be the zeros of some $f(x) \in \mathbb{Z}[x]$ of degree $n$. If there exists an integer $b$ such that $f(b)$ is prime, $f(b-1) \neq 0$ and

$$
\Re\left(\beta_{i}\right)<b-1 / 2 \quad \text { for } 1 \leq i \leq n,
$$

then $f(x)$ is irreducible over $\mathbb{Q}$.

We consider the beta-polynomials of $\Delta(1 / q)$ and $\Delta(1 / q+)$. Denote them by $f_{q}^{l}(x)$ and $f_{q}^{u}(x)$ respectively, and take $b=2$. Then $f_{q}^{l}(1)=f_{q}^{u}(1)=-1$. If $q \geq 3$ then $\Delta(1 / q)<3 / 2$, and if $q \geq 4$ then $\Delta(1 / q+)<3 / 2$. Since beta-numbers are Perron numbers [11], condition (4) holds for lower (resp. upper) self-Christoffel numbers whenever $q \geq 3$ (resp. $q \geq 4$ ). We also note that

$$
f_{q}^{l}(2)=2^{q-1}-1, \quad f_{q}^{u}(2)=2^{q}-3 .
$$

Now one can say the following: 
1. If there exist infinitely many Mersenne primes, then $f_{q}^{l}(x)$ is irreducible for infinitely many $q \geq 3$.

2. If there exist infinitely many primes of the form $2^{n}-3$, then $f_{q}^{u}(x)$ is irreducible for infinitely many $q \geq 4$.

Using a computer the author checked the irreducibility of beta-polynomials of self-Christoffel numbers. To be more precise, suppose $\alpha=p / q+b-1$, where $1 \leq p<q \leq 200, \operatorname{gcd}(p, q)=1$ and $1 \leq b \leq 150$. For given $1 / 2 \leq$ $p / q \leq 199 / 200$, the irreducibility of beta-polynomials of $\Delta(\alpha)$ and $\Delta(\alpha+)$ was checked for $1 \leq b \leq 150$. When some beta-polynomial is reducible, the case was recorded as " $(p, q, b)$ " in a row. As a result, a table was obtained, which comprises 5642 (resp. 3422) reducible cases for lower (resp. upper) self-Christoffel numbers. This is too huge to be included here. We refer to [8] instead. For example, if $\alpha=6 / 11+b-1$ then the beta-polynomial of $\Delta(\alpha)$ is irreducible for all $b=1,2,4 \leq b \leq 150$, and the beta-polynomial of $\Delta(\alpha+)$ is irreducible for all $2 \leq b \leq 150$. On the other hand, if $\alpha=4 / 9+b-1$ then the beta-polynomials of both $\Delta(\alpha)$ and $\Delta(\alpha+)$ are irreducible for all $1 \leq b \leq 150$. We will use this table below implicitly.

Suppose $\alpha=p / q+b-1$. If we follow the proof of the Theorem described in Section 3, then we can find effectively the smallest possible constant $B$ for which $b \geq B$ implies that the beta-polynomial of $\Delta(\alpha)$ or $\Delta(\alpha+)$ is irreducible. This procedure is demonstrated in the next example.

EXAmple 4.2. Let $\alpha=4 / 7+b-1$ and let us find $B$ for the upper self-Christoffel number $\Delta(\alpha+)$. First we must determine $B_{1}$ so that the Mahler measure of $g$ is less than $\theta_{0}$ given in Theorem 2.1. One can see that it is enough that

$$
\left(\frac{B_{1}+\sqrt{B_{1}^{2}+4 B_{1}}}{2 B_{1}}\right)^{5}<\theta_{0} .
$$

So $B_{1} \geq 17$. Second we also find $B_{2}$ which contradicts inequality (3). Computation shows that $B_{2} \geq 11$. Let $B_{3}=\max \left\{B_{1}, B_{2}\right\}=17$. Then for $b \geq B_{3}$ the beta-polynomial of $\Delta((4 / 7+b-1)+)$ is irreducible. One readily notes that the constant $B_{3}$ works not only for $4 / 7$ but for all $1 / 7,2 / 7, \ldots, 6 / 7$. To find the smallest $B$ instead of $B_{3}$, we consult the table of [8]. It suffices to check whether or not $(1,7, b),(2,7, b), \ldots,(6,7, b)$ for $1 \leq b \leq 16$ are on the list. But none of them is, even for $1 \leq b \leq 150$. Now we can state the following (i.e., in this case the beta-polynomial of $\Delta(\alpha+)$ is irreducible for every $b \geq B=1)$ :

For any integer $p \geq 1$ which is not a multiple of 7 , the beta-polynomial of $\Delta(p / 7+)$ is irreducible.

Some calculations tempt us to conjecture that all lower self-Christoffel numbers are Pisot numbers. But this is false as explained below. 
Flatto et al. [5] considered the dynamical zeta-function

$$
\zeta_{\beta}(z)=\exp \left(\sum_{n=1}^{\infty} \frac{P_{n}}{n} z^{n}\right)
$$

for the $\beta$-transformation $T_{\beta}$, where $P_{n}$ is the number of fixed points of $T_{\beta}^{n}$. They wrote it in terms of the $\beta$-expansions of 1 :

1. If $\beta$ is not a simple beta-number with $d_{\beta}(1)=e_{1} e_{2} \cdots$, then

$$
\zeta_{\beta}(z)=\frac{1}{1-\sum_{i=1}^{\infty} e_{i} z^{i}} .
$$

2. If $\beta$ is a simple beta-number with $d_{\beta}(1)=e_{1} \cdots e_{n}$, then

$$
\zeta_{\beta}(z)=\frac{1-z^{n}}{1-\sum_{i=1}^{n} e_{i} z^{i}} .
$$

In both cases, $\zeta_{\beta}(z)$ is meromorphic in the open unit disk. It has a simple pole at $z=1 / \beta$ and no other pole in $\{z:|z| \leq 1 / \beta\}$. Then $M(\beta)$ was defined by the second smallest modulus of the poles of $\zeta_{\beta}(z)$. If no pole other than $1 / \beta$ exists in the open unit disk, then $M(\beta)=1$. The main interest was the behavior of the function $M(\beta)$. The next theorem hints at the possible abundance of non-Pisot lower self-Christoffel numbers.

Theorem $4.3([5])$. There exists $\varepsilon>0$ such that $M(\beta)<1$ for every $\beta \in(1,1+\varepsilon)$.

In particular, if the Mersenne prime conjecture is true then there are infinitely many non-Pisot lower self-Christoffel numbers. There still remains a question in this direction.

Question 1. Let $p / q$ be a fixed rational with $0<p \leq q$ and $\operatorname{gcd}(p, q)$ $=1$. If $\alpha=b-1+p / q$ for $b \in \mathbb{N}$, is $\Delta(\alpha)$ eventually a Pisot number as $b$ increases?

Together with the Theorem, an affirmative answer to Question 1 would produce many concrete examples of irreducible Pisot type beta-substitutions. See [1] for details.

While our main interest in this paper is irreducible beta-polynomials, Boyd [2] focused on reducible beta-polynomials. Specifically, he dealt with the situation where $\beta$ is a Pisot number. It is well known that every Pisot number is a beta-number [16]. Let $\beta$ be a Pisot number and $f(x)$ be the $\beta$-polynomial. Now we suppose that $f(x)=g(x) h(x)$ is a nontrivial factorization over $\mathbb{Q}$, where $h(x)$ is the minimal polynomial of $\beta$. Boyd called $g(x)$ the complementary factor. Although extensive calculations made some mathematicians suspect that $g(x)$ should be a product of cyclotomic polynomials, Boyd showed by systematic computation that there exist many Pisot 
numbers $\beta$ for which their complementary factors are noncyclotomic or even nonreciprocal.

Motivated by Boyd's work, the author checked the complementary factors of all reducible cases listed in [8] for both lower (5642) and upper (3422) self-Christoffel numbers. Surprisingly enough, all the complementary factors are (either cyclotomic polynomials or) products of cyclotomic polynomials.

For lower self-Christoffel numbers in [8], all irreducibles of the complementary factors are among the $\Phi_{n}$ with $n=2,3,4,5,6,7,8,9,10,12,14,15$, $16,18,20,21,22,24,26,28$, or 30 , where $\Phi_{n}$ is the $n$th cyclotomic polynomial. For upper self-Christoffel numbers in [8], all irreducibles of the complementary factors are among the $\Phi_{n}$ with $n=1,2,3,4,5,6,8,9,10,12$, or 14 .

A natural question arises.

Question 2. For $\beta$ a lower or an upper self-Christoffel number, if the $\beta$ polynomial is reducible, is its complementary factor a product of cyclotomic polynomials?

\section{References}

[1] V. Berthé and A. Siegel, Tilings associated with beta-numeration and substitutions, Integers 5 (2005), no. 3, A2, 46 pp.

[2] D. W. Boyd, On beta-expansions for Pisot numbers, Math. Comp. 65 (1996), 841860.

[3] J. W. Cannon and Ph. Wagreich, Growth functions of surface groups, Math. Ann. 293 (1992), 239-257.

[4] D. P. Chi and D. Y. Kwon, Sturmian words, $\beta$-shifts, and transcendence, Theoret. Comput. Sci. 321 (2004), 395-404.

[5] L. Flatto, J. C. Lagarias and B. Poonen, The zeta function of the beta transformation, Ergodic Theory Dynam. Systems 14 (1994), 237-266.

[6] D. Y. Kwon, A devil's staircase from rotations and irrationality measures for Liouville numbers, preprint, 2004, http://arxiv.org/abs/0709.1642.

[7] —, Beta-numbers whose conjugates lie near the unit circle, Acta Arith. 127 (2007), $33-47$.

[8] —, Table of reducible beta-polynomials of lower and upper self-Christoffel numbers, http://math.yonsei.ac.kr/doyong/paper/reducible.pdf.

[9] P. Lakatos, On zeros of reciprocal polynomials, Publ. Math. Debrecen 61 (2002), 645-661.

[10] D. H. Lehmer, Factorization of certain cyclotomic functions, Ann. of Math. (2) 34 (1933), 461-479.

[11] D. A. Lind, The entropies of topological Markov shifts and a related class of algebraic integers, Ergodic Theory Dynam. Systems 4 (1984), 283-300.

[12] M. Lothaire, Algebraic Combinatorics on Words, Cambridge Univ. Press, New York, 2002.

[13] W. Parry, On the $\beta$-expansions of real numbers, Acta Math. Acad. Sci. Hungar. 11 (1960), 401-416.

[14] G. Pólya and G. Szegö, Problems and Theorems in Analysis II, Springer, New York, 1976. 
[15] A. Rényi, Representations for real numbers and their ergodic properties, Acta Math. Acad. Sci. Hungar. 8 (1957), 477-493.

[16] K. Schmidt, On periodic expansions of Pisot numbers and Salem numbers, Bull. London Math. Soc. 12 (1980), 269-278.

[17] C. J. Smyth, On the product of the conjugates outside the unit circle of an algebraic integer, ibid. 3 (1971), 169-175.

Department of Mathematics

Yonsei University

134 Shinchon-dong, Seodaemun-gu

Seoul 120-749, Republic of Korea

E-mail: doyong@yonsei.ac.kr

Received on 21.3.2007

and in revised form on 12.9.2007 